\title{
Features and Analyses of W7-X Cryostat System FE Model
}

\author{
$\underline{\text { Paul van Eeten }}^{\mathrm{a}}$, Torsten Bräuer ${ }^{\mathrm{a}}$, Victor Bykov ${ }^{\mathrm{a}}$, Andre Carls $^{\mathrm{a}}$, Joris Fellinger ${ }^{\mathrm{a}}$, J.P. Kallmeyer ${ }^{\mathrm{a}}$ \\ ${ }^{a}$ Max-Planck-Institut für Plasmaphysik, Wendelsteinstrasse 1, D-17491 Greifswald, Germany
}

The Wendelstein 7-X stellarator is presently under construction at the Max-Planck-Institute for Plasma Physics in Greifswald with the goal to verify that a stellarator magnetic confinement concept is a viable option for a fusion power plant.

The main components of the W7-X cryostat system are the plasma vessel (PV), outer vessel (OV), ports, thermal insulation, vessel supports and the machine base (MB). The main task of the cryostat system is to provide an insulating vacuum for the cryogenic magnet system while allowing external access to the PV through ports for diagnostic, supply and heating systems.

The cryostat is subjected to different types of loads during assembly, maintenance and operation. This ranges from basic weight loads from all installed components to mechanical, vacuum and thermal loads. To predict the behaviour of the cryostat in terms of deformations, stresses and support load distribution a finite element (FE) global model has been created called the Global Model of the Cryostat System (GMCS).

A complete refurbishment of the GM CS has been done in the last 2 years to prepare the model for future applications. This involved a complete mesh update of the model, an improvement of many model features, an update of the applied operational loads and boundary conditions as well as the creation of automatic post processing procedures.

Currently the GMCS is used to support several significant assembly and commissioning steps of W7-X that involve the cryostat system, e.g. the removal of temporary supports beneath the MB, transfer of the PV from temporary to the final supports and evacuation of the cryostat. In the upcoming months the model will be used for further support of the commissioning of W7-X which includes the first evacuation of the PV.

Keywords: structural analysis, W7-X, cryostat, finite element, stellarator, commissioning, metrology.

\section{Introduction}

The WENDELSTEIN 7-X (W7-X) stellarator experiment is currently under construction at the MaxPlanck-Institute for Plasma Physics in Greifswald. Design, manufacturing and assembly of the main structural components have finished and focus is now lying on commissioning of the basic device [1] and manufacturing and assembly of diagnostics and peripheral systems.

The W7-X cryostat mainly consists of the Outer Vessel (OV), the Plasma Vessel (PV), 254 ports with bellows that connect the vessels, and the thermal insulation (Figure 1). The OV, PV and ports enclose the volume of the cryostat. The cryostat provides an insulating vacuum for the W7-X cryogenic magnet system (MS) which is enclosed by the cryostat while allowing external access to the PV through ports for diagnostic, supply and heating systems. The cryostat is positioned on the machine base (MB) through PV- and $\mathrm{OV}$-supports. The PV-supports consist of horizontal and vertical supports. These supports can also be used to adjust the position of the PV relative to the OV.

The cryostat and the MS have separate and independent supports. The MB forms the only interface between the MS and the cryostat. As the only interaction is basically dead weight deformation of the MB, the structural behaviour of both MS and cryostat can be assessed independently.

Both for the cryostat and the MS finite element (FE) global models have been created to be able to analyse the global behaviour of both systems with respect to displacement, stresses and load distribution ([2], [3]).

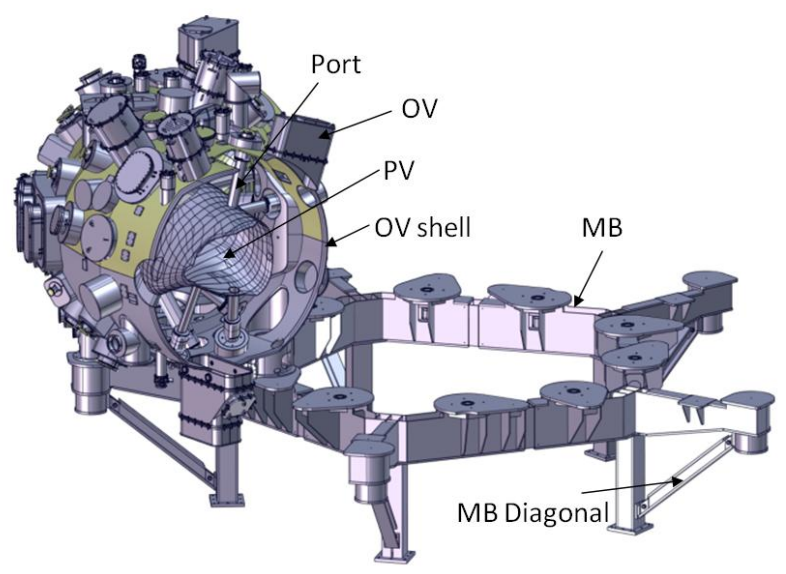

Figure 1 W7-X cryostat: 1 of 5 modules incl. MB

In 2014 the W7-X has started commissioning of the basic machine in preparation of achieving first plasma in mid-2015 [1]. This paper focuses on the verification of the global model of the cryostat system (GMCS) using measurements performed during the first commissioning steps.

\section{FE Analyses with GMCS}

\subsection{Model revision}

To prepare the GMCS for the commissioning phase the model has been revised compared to the status as 
presented in [3]. The revision included the following changes:

- Introduction of port reduction for PV and OV (from 299 to 254 ports).

- Updated geometries and improved mesh and for vessels, ports and PV supports incl. updated shell element type.

- Introduction of contact and compressiononly elements at PV horizontal and vertical supports [4].

- $\quad$ Port bellows and the torus hall (TH) floor simulation and using super elements.

- Update of load cases incl. update of thermal load distribution.

- Creation of a large set of post processing macros for extracting the most relevant structural output from the GMCS FE results.

Table 1. GMCS FE Model summary:

\begin{tabular}{|l|l|}
\hline Finite elements & $1,500,000$ \\
\hline Nodes & $\sim 820,000$ \\
\hline Degrees of freedom & $\sim 2,500,000$ \\
\hline Active contact interfaces & 35 \\
\hline Linear material types & 4 \\
\hline Mass of simulated system & $\sim 330$ metric tons \\
\hline
\end{tabular}

\subsection{GMCS Load Cases}

During operation, the cryostat system of the W7-X stellarator is subjected to different types of loads. The loads cover weight, thermal, electro-magnetic and vacuum loads. In addition a set of abnormal cases is taken into account to determine the impact of certain fault scenarios like $\mathrm{He}$ - or water-pipe leakage and subsequent over-pressure in the vessels or eddy current loads induced by a fast shutdown of the main magnet system. Table 2 shows a selection of typical load cases (total 21) as covered by the GMCS. For all load cases the weight of the MS is included. In addition weight and active loads for KIP, diagnostics and peripheral systems is included in the GMCS.

Table 2. GMCS load cases

\begin{tabular}{|c|c|c|c|c|c|c|}
\hline \multirow[t]{2}{*}{ LC\# } & \multicolumn{2}{|c|}{$\begin{array}{c}\text { Pressure } \\
\text { (bar) }\end{array}$} & \multirow[t]{2}{*}{$\mathrm{PV}^{\circ} \mathrm{C}$} & \multirow[t]{2}{*}{$\mathrm{OV}^{\circ} \mathrm{C}$} & \multirow[t]{2}{*}{$\begin{array}{c}\text { Probab } \\
\text { ility }\end{array}$} & \multirow[t]{2}{*}{ Explanation } \\
\hline & in PV & in $0 \mathrm{~V}$ & & & & \\
\hline 1 & 1 & 1 & RT & RT & & Assembly phase \\
\hline 2 & 0 & 0 & 150 & Gradient* & & Baking \\
\hline $3 a$ & 0 & 0 & $\mathrm{RT}^{* *}$ & RT & & $\begin{array}{l}\text { Normal } \\
\text { operation }\end{array}$ \\
\hline $3 b$ & 0 & 0 & $80^{* *}$ & RT & & $\begin{array}{l}\text { Normal } \\
\text { operation / } \\
\text { warm PV }\end{array}$ \\
\hline 4 & 1 & 0 & RT & RT & & $\begin{array}{l}\text { Maintenance in } \\
\text { PV }\end{array}$ \\
\hline 5 & 0 & 1 & RT & RT & & $\begin{array}{l}\text { Maintenance in } \\
\text { OV }\end{array}$ \\
\hline $\mathrm{AN}-2 \mathrm{a}$ & 1,1 & 0,0 & 150 & $\underset{*}{\text { Gradient }}$ & likely & \multirow{4}{*}{$\begin{array}{l}\text { Overpressure in } \\
\text { OV or PV during } \\
\text { baking scenarios }\end{array}$} \\
\hline$A N-2 b$ & 1,2 & 0,0 & 150 & $\underset{* *}{\text { Gradient }}$ & unlikely & \\
\hline $\mathrm{AN}-2 \mathrm{c}$ & 0,0 & 1,2 & 150 & $\underset{* *}{\text { Gradient }}$ & unlikely & \\
\hline $\mathrm{AN}-2 \mathrm{e}$ & 0,0 & 1,0 & 150 & $\underset{* *}{\operatorname{Gradient}}$ & likely & \\
\hline
\end{tabular}

\begin{tabular}{|c|c|c|c|c|l|l|}
\hline AN-3i-2 & 1,7 & 0,0 & 80 & RT & $\begin{array}{l}\text { very } \\
\text { unlikely }\end{array}$ & $\begin{array}{l}\text { Overpressure in } \\
\text { PV normal } \\
\text { operation incl. } \\
\text { eddy current } \\
\text { loads }\end{array}$ \\
\hline AN-7a & 1,0 & 0,0 & RT & -10 & unlikely & $\begin{array}{l}\text { Large liq. He leak } \\
\text { in cryostat }\end{array}$ \\
\hline AN-8b & 0,0 & 0,0 & RT & 50 & $\begin{array}{l}\text { very } \\
\text { unlikely }\end{array}$ & $\begin{array}{l}\text { Warm OV due to } \\
\text { external heat } \\
\text { source (e.g. fire) }\end{array}$ \\
\hline
\end{tabular}

*: For baking the outer vessel will have a temperature gradient between the hot components $(150 \mathrm{degC}$ at port dome plate interface) and the OV components at RT.

**: For long pulse operation it still needs a detailed assessment on the expected PV temperature.

\section{GMCS verification during $\mathrm{W} 7-\mathrm{X}$ commissioning}

\subsection{Metrology}

For verification of the GMCS results geometrical measurements [5] were carried out during load changes forced by the removal of temporary supports and evacuation as well as venting of cryostat.

The principal approach of these measurements accompanying the load changes was:

- Installation of targets at positions discussed between metrology and engineering group. Usually RFIs (reflector for fixed installation) are used for targets.

- Orientation of the measurement system to the global W7-X co-ordinate system which is represented by 128 reference points fixed on the TH walls.

- Initial reference measurement of target coordinates before start of load change.

- Frequent measurements of target position during load transients incl. quasi-real time data transfer to the responsible engineers.

The most appropriated measurement tools for these tasks are Laser-Trackers (LT). The metrology group at IPP owns LT from Leica with a guaranteed measurement uncertainty of $\pm 10 \mu \mathrm{m} / \mathrm{m} \pm 25 \mu \mathrm{m}$ for a $3 \mathrm{D}$ co-ordinate. For W7-X, i.e. inside the TH $\left(32 \times 30 \times 15 \mathrm{~m}^{3}\right)$ including effects like air condition and daily assembly work, the measurement uncertainty can be estimated by $0.3 \mathrm{~mm}$ for the global position of a target and $0.1 \mathrm{~mm}$ for repetition measurements. To achieve these accuracies the atmospheric conditions within the TH play a significant role. It was required to find a compromise between keeping stable temperature conditions through air conditioning and minimizing air flow in the TH. The LT are sensitive to temperature gradients as the layers of different air densities influence the laser path. The turbulences due to air flow when the air conditioning is on increases the noise on the measured values. The compromise chosen in many cases was to switch off the air condition temporary for the most sensitive measurements resulting in a gradual temperature 
increase in the $\mathrm{TH}$ warming $\mathrm{W} 7-\mathrm{X}$ and subsequent displacements.

The removal of the temporary supports of MB (1 support for each of the 5 modules) took place sequentially. Thus, monitoring of removal process could be realized by one LT only. To get the most accurate results $(0.1 \mathrm{~mm}$ for repetition measurements) the LT was not moved during the removal process of a particular support. At each support location 5 targets on the MB, 2 on OV and 2 on $\mathrm{PV}$ were obtained and co-ordinates were provided to the engineering group for deeper analysis.

Evacuation and venting of the cryostat were monitored using 25 targets on OV and 17 targets linked to the PV through supply ports. The locations of the targets had been preselected by the engineering group on conditions that approx. $70 \%$ of the points are at locations with high and $30 \%$ of the point at locations with only small deformations due to vacuum load changes. An additional requirement was to have the points distributed on all 5 modules as symmetric as possible. In reality those conditions could be fulfilled only partially using points on the upper hemisphere of the cryostat. The main restrictions were a limited number of LTs available and very restricted access due to intensive assembly activities close to the cryostat. Finally 3 LTs were used for the global survey of the cryostat whereas one additional LT was used to monitor a special sensitive $\mathrm{OV}$ location in real-time during the entire evacuation/venting process.

\subsection{Removal of temporary MB supports}

As the assembly of the peripheral systems continued it was eventually required to remove all temporary supports (TS) that were in place to support the assembly of the basic machine. Among several temporary supports for the magnet and cryostat system 5 TS were installed below the 5 outer MB supports. Removal of the TS would induce sag of several $\mathrm{mm}$ in the outer support beams of the MB resulting in sag of the supported OV and PV. This would introduce additional inaccuracies in the position of in-vessel components already installed in the PV (wall panels, diagnostic etc.). To counteract this sag, it was decided to hydraulically lift the outer support up as such that, after fixation of the diagonal beam (Figure 1) and release of the applied hydraulic load, the whole system would return to the same position as with the TS installed. The GMCS was used to predict the required hydraulic load and vertical displacement. The upward displacement was performed with a disconnected diagonal beam. At the lifted position the diagonal was fixed by reaming the interface and introducing shear bolts. After removal of the hydraulic load the stiffened system was lowered to its final position. Fig. 2 shows a comparison for a typical module between the prediction and the measured behaviour. The system stiffness (gradient), both with and without active diagonal beam showed only a $\sim 20 \%$ deviation between prediction and measurement. In all modules larger deviations where found in predicting the load at the initial position $(0 \mathrm{~mm})$ as being carried by the TS. Since all MB supports together create an over-determined system this is explainable by inaccurate position of the TS at the initial installation of the MB. This discrepancy of the initial load made it necessary to increase the hydraulic load and subsequent vertical lift. The increased load compensated the discrepancy and minimized final sag in comparison with the target position but resulted in a higher load within the MB outer support and diagonal.

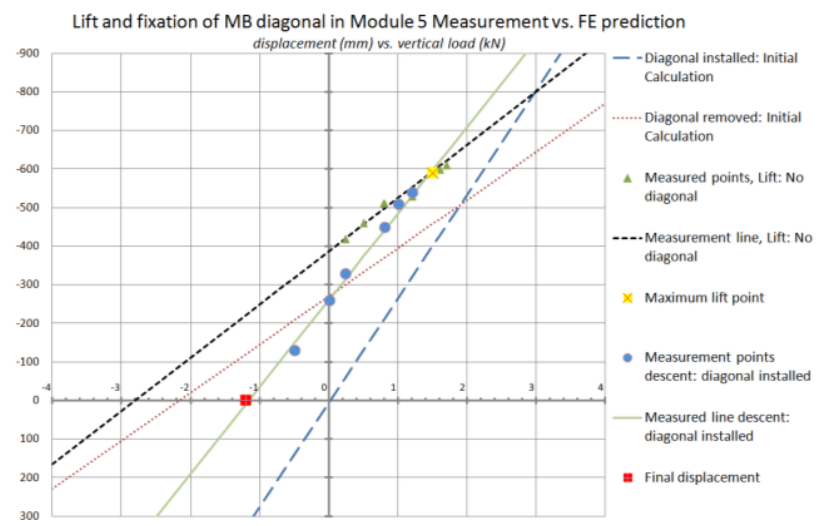

Figure 2 Prediction and measurement of $\mathrm{MB}$ diagonal fixation

\subsection{Cryostat evacuation}

The first major commissioning step for W7-X [1] concerned the evacuation of the cryostat with the goal to achieve $10^{-5} \mathrm{mbar}$ as a prerequisite for starting cool down of the cryostat and magnet system. The evacuation introduces vacuum loads on the OV, PV and ports causing the structure to deform. The cryostat evacuation corresponds to load case 4 as shown in Table 2 . In addition to the post processing macros for extracting the predicted structural values (displacements / stresses) a matlab tool was developed to generate real-time stress values from the raw PV strain gauge data being delivered to the central data archive permanently.

Too verify the structural integrity of the cryostat during the evacuation process, several accompanying measurements were performed:

1. LT measurements of 42 reference points on OV shell, domes and supply ports

2. Monitoring of PV strain gauges

3. Monitoring of PV pendulum support load.

4. Horizontal PV support loads.

5. Deformation of large rectangular and oval port bellows.

Figure 3 shows the evolution of the cryostat pressure and $\mathrm{TH}$ ambient air temperature during the first evacuation of the cryostat. Two pressure plateaus at 700 and 300 mbar were scheduled to provide time for different measurements of the cryostat structure at a constant pressure level. Based on these measurements it was decided to continue evacuation. To aid acoustic monitoring during evacuation loud acoustic sources (mainly TH air conditioning system) were shut down during the time of first evacuation. This resulted in an increase of the TH temperature as seen in Figure 3. The $\mathrm{TH}$ air condition system was activated again after roughly $60 \mathrm{hrs}$ of pumping time resulting in a fast decrease of $\mathrm{TH}$ temperature. 
Cryostat evacuation

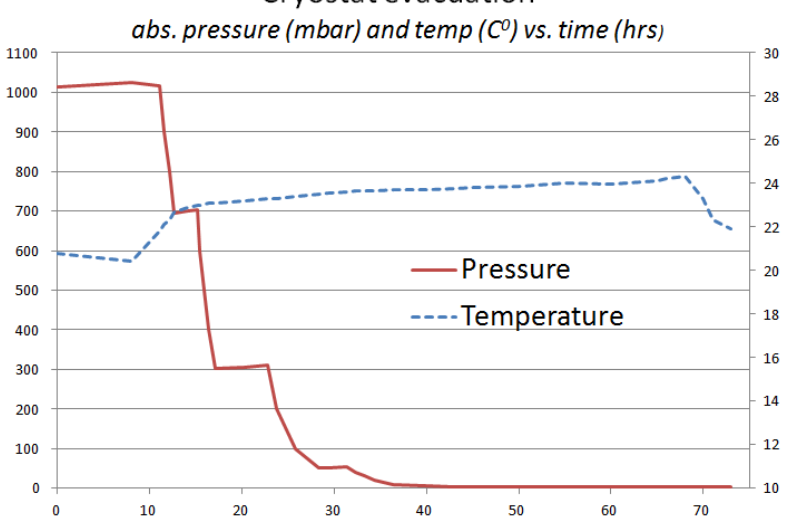

Figure 3 Cryostat pressure and $\mathrm{TH}$ temperature during cryostat evacuation

The response of the cryostat and its supports to the vacuum loads is basically linear. This was confirmed by the results of the PV strain gauge, PV support load and port bellows deformation measurements. All of these measurements showed good agreement with the behaviour as predicted by the GMCS in these locations. The 42 reference points as tracked during evacuation showed for the larger part $(\sim 70 \%)$ good agreement with the prediction from the GMCS. About 10 points, both on $\mathrm{OV}$ and on supply ports showed stronger deviations. Figure 4 includes the prediction and the measured results for two typical OV points and two supply ports. It is clearly seen that displacement measurements at pressures below 50 mbar show larger fluctuations.

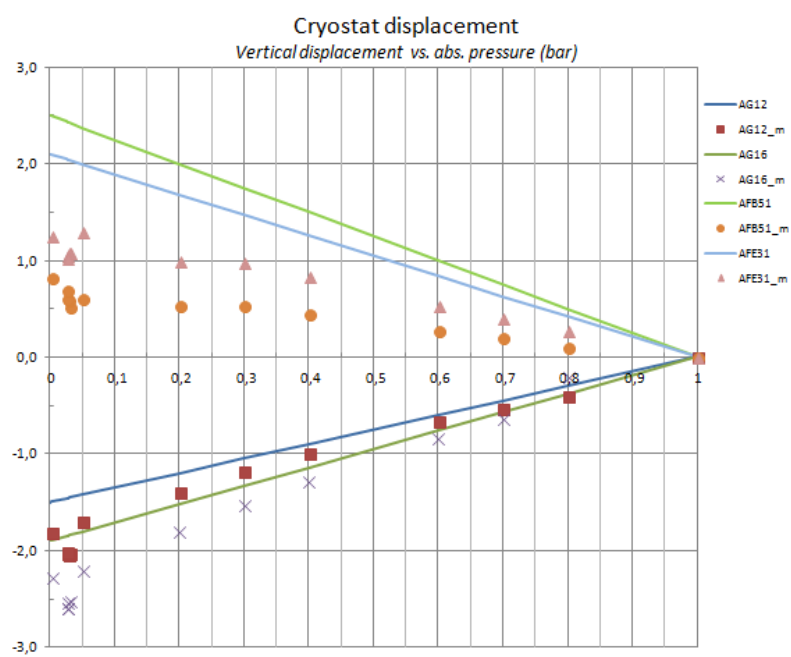

Figure 4 Typical results of reference point measurements

As seen in Figure 3 the pressure reduction rate decreases considerably below $\sim 50$ mbar. The measurements were taken at longer intervals thus becoming more sensitive to the ambient conditions in the TH. The "kink" in the measurements for $\mathrm{P}<50 \mathrm{mbar}$ are caused by the long measurement interval and the fast cool down of the TH after activation of the air conditioning system.

The measurements on PV strain gauges, OV/Port displacements, PV support loads and port bellows deformations during the evacuation process provided a solid basis for judging the structural integrity of the W7$\mathrm{X}$ cryostat and for a first validation of the GMCS.

\section{Conclusion}

The different measurements confirmed the validity of the predictions done by the GMCS. The LT measurements provided some difficulties with respect to reference point measurement fluctuations. This is most probably mainly due to the non-static ambient conditions in the $\mathrm{TH}$ and longer measurement intervals during the evacuation campaign. The verification did not show any discrepancies that indicate major structural issues for the cryostat. The GMCS is predominantly used for deformation analyses. The results show that the mesh size (Table 1) provides a good approximation of the system stiffness and its support behaviour whilst keeping a lean model for reduced calculation time. The GMCS does not directly allow for local stress analysis of welded interfaces and other details. The GMCS can provide nodal output (displacement fields or nodal forces and moments) to be used in analytical tools or sub model boundary conditions.

After the first evacuation of the cryostat the project management has given permission to enter the PV (within the evacuated cryostat) for continuing in-vessel assembly tasks. The next commissioning step covers cool down of structures within the cryostat (e.g. thermal insulation, magnet- and magnet support system) which should not impose any additional load onto the cryostat. The next commissioning step of direct relevance to the GMCS is the evacuation of the PV. This will again be closely monitored by accompanying measurements and verification of the GMCS predictions.

\section{Acknowledgments}

The author would like to thank all contributors to the GMCS verification including metrology, quality management, machine instrumentation, $\mathrm{CoDaC}$, engineering and assembly. This work has been carried out within the framework of the EUROfusion Consortium and has received funding from the European Union's Horizon 2020 research and innovation program under grant agreement number 633053. The views and opinions expressed herein do not necessarily reflect those of the European Commission.

\section{References}

[1] H.-S. Bosch et al., "Experience with the commissioning of the superconducting stellarator Wendelstein 7-X", to be presented at SOFT2014

[2] V. Bykov et al., "Specific Features of Wendelstein 7-X Structural Analyses", IEEE transactions on plasma science, vol. 42, no. 3, march 2014

[3] A. Tereshchenko et al., "FE simulation of the Wendelstein 7-X cryostat system", Fusion Engineering and Design 84 (2009) 1833-1837

[4] B. Missal et al., "Pendulum support of the W7-X plasma vessel: Design, tests, manufacturing, assembly, critical aspects, status", Fusion Engineering and Design 89 (2014) 1820-1825

[5] T. Bräuer et al., "On the accuracy of port assembly at Wendelstein 7-X", Fusion Engineering and Design 88 (2013) 721-724 